\title{
Treatment Free Intervals after Subsequent Chemotherapy Lines in Recurrent Ovarian Cancer
}

\author{
Hanna Sallinen ${ }^{1}$, Vivi Rintanen ${ }^{1,2}$, Leea Keski-Nisula ${ }^{1,2}$, Maarit Anttila1 \\ ${ }^{1}$ Department of Gynecology and Obstetrics, Kuopio University Hospital, Kuopio, Finland \\ ${ }^{2}$ Department of Health Sciences, Clinical Medicine, University of Eastern Finland, Kuopio, Finland \\ Email: ^hanna.sallinen@kuh.fi
}

How to cite this paper: Sallinen, H., Rintanen, V., Keski-Nisula, L. and Anttila, M. (2021) Treatment Free Intervals after Subsequent Chemotherapy Lines in Recurrent Ovarian Cancer. Journal of Cancer Therapy, 12, 346-357.

https://doi.org/10.4236/jct.2021.126031

Received: April 24, 2021

Accepted: June 6, 2021

Published: June 9, 2021

Copyright $\odot 2021$ by author(s) and Scientific Research Publishing Inc. This work is licensed under the Creative Commons Attribution International License (CC BY 4.0).

http://creativecommons.org/licenses/by/4.0/

\begin{abstract}
Aim: Despite recent advances in the treatment of ovarian cancer, recurrence of the disease is still frequent. This study evaluated whether multiple lines of chemotherapy have impact on overall survival (OS), progression free survival (PFS) or on treatment free intervals (TFIs) after serial chemotherapy lines in recurrent settings. Methods: A total of 189 patients with ovarian cancer (including fallopian tube and primary peritoneal cancer), who were treated in Kuopio University Hospital in Finland during 2009-2014, were enrolled. The medical files of these patients were retrospectively reviewed. Results: Median OS and PFS were significantly higher at the time of the first relapse compared to subsequent relapses $(\mathrm{p}<0.001)$. TFIs shortened significantly after the first relapse $(\mathrm{p}<0.001)$. The differences in TFIs were also seen when comparing platinum sensitive, semi-sensitive and platinum resistant patients. The total amount of TFI times during the whole follow-up time was significantly reduced in those patients that received at least one form of aggressive care at the end of life ( $p=0.004)$. Conclusions: Ovarian cancer patients received often multiple lines of chemotherapy. TFIs after subsequent chemotherapy lines decreased during the disease course. More efforts should be taken to avoid unnecessary and ineffective treatments especially in recurrent phase of the disease.
\end{abstract}

\section{Keywords}

Ovarian Cancer, Chemotherapy, Treatment Free Interval, Overall Survival, Progression Free Survival, Aggressive Care

\section{Introduction}

In Nordic countries, the average 5-year overall survival rate in ovarian cancer is 
$45 \%$ [1]. Although all efforts have been made in optimization of the treatments, OS has not increased in the last 25 years [2]. Because of the chronic nature of the disease, patients with ovarian cancer are often treated with serial lines of chemotherapy during their disease [3].

It has been shown in several advanced cancers that PFS decreases after each subsequent chemotherapy line [4]. TFIs might be another way to assess cancer progression and patient well-being. TFI is the time between the end of the last regimen to the beginning of the next regimen. It describes the time when patients are without active cancer-targeted medication. In clinical trials the number of prior chemotherapy lines is often an eligibility criterion since prior chemotherapy lines impact the likelihood of response to further treatments [5]. Despite that, most studies focus on patients with second or third line of chemotherapy. There is a need for studies beyond third line settings. Therefore, we evaluated OS, PFS and TFI rates also in more heavily treated patients.

Studies where TFI has been used to measure success of multiple chemotherapy lines during the whole follow-up time of ovarian cancer patients, are sparse [3] [6]. We have recently reported our experience that a majority of the patients receives aggressive care in the last 30 days of their lives according to the standards of The National Quality Forum [7]. In the present study, we studied how TFI was associated with aggressive care at the end of life.

\section{Methods}

This retrospective study investigated patients who had been treated due to ovarian, fallopian tube or primary peritoneal cancer between years 2009-2014 in Kuopio University Hospital in Finland. A total of 189 patients were included in this cohort. Fallopian tube and primary peritoneal cancers are included since they are clinically similar in presentation and are treated similarly. A patient was selected from the medical register of our hospital if she had received primary treatment for her cancer, and if she had also been followed up in our institution. Characteristics of the patients are summarized in Table 1. The medical files of these patients were retrospectively reviewed. Data were abstracted and entered to a computerized database. Abstracted data included age, the date of diagnosis, stage, grade and histology of cancer, number and dates of relapses, all cancer treatments and date and location of death.

The term of recurrence/relapse was defined clinically, i.e. physical evidence upon imaging or examination and/or a rise in CA-125 marker evaluated by physicians. Not responding to treatment or progression during treatment was interpreted as treatment-resistant disease, not relapse. OS was calculated from the day of diagnosis and then from each subsequent documented relapse to death. PFS was calculated from the day of diagnosis to the first relapse and then from one relapse to the subsequent one or death. TFI was calculated from the end of one chemotherapy-regimen to the start of the next regimen. The aggressive care was defined according to the standards outlined by the National Quality Forum; 
Table 1. Characteristics of the patients, $\mathrm{n}=189$.

\begin{tabular}{|c|c|}
\hline Characteristic $(n=189)$ & $n(\%)$ \\
\hline Age median (range) & $64(26-94)$ \\
\hline \multicolumn{2}{|l|}{ Histology } \\
\hline Serous $^{\dagger}$ & $133(70)$ \\
\hline Mucinous & $9(5)$ \\
\hline Endometroid & $17(9)$ \\
\hline Clear cell & $11(6)$ \\
\hline Histology unknown & $10(5)$ \\
\hline Other ${ }^{\ddagger}$ & $9(5)$ \\
\hline \multicolumn{2}{|l|}{ Stage } \\
\hline I & $23(12)$ \\
\hline II & $8(4)$ \\
\hline III & $86(46)$ \\
\hline IV & $65(34)$ \\
\hline Not documented & $7(4)$ \\
\hline \multicolumn{2}{|l|}{ Grade } \\
\hline High grade & $154(82)$ \\
\hline Low grade & $8(4)$ \\
\hline Not documented & $27(14)$ \\
\hline \multicolumn{2}{|l|}{ Diagnostics } \\
\hline Primary cytoreductive surgery & $131(70)$ \\
\hline Secondary cytoreductive surgery & $29(16)$ \\
\hline Diagnostic laparoscopy & $5(3)$ \\
\hline Biopsy & $11(6)$ \\
\hline Imaging & $12(6)$ \\
\hline Not documented & $1(1)$ \\
\hline Operated patients Residual tumor $(\mathrm{cm})$ & $160(85)$ \\
\hline none & $76(48)$ \\
\hline$<1$ & $28(17)$ \\
\hline$\geq 1$ & $48(30)$ \\
\hline Not documented & $8(5)$ \\
\hline \multicolumn{2}{|l|}{ Tumor recurrence } \\
\hline Recurrent & $116(61)$ \\
\hline Not recurrent & $60(32)$ \\
\hline Resistant to treatment & $13(7)$ \\
\hline Death during the follow-up & $104(55)$ \\
\hline Median follow-up time (months) & $35(0-189)$ \\
\hline
\end{tabular}

${ }^{\dagger} 15$ (7.9\%) Fallopian tube, 11 (5.8\%) Primary peritoneal; ${ }^{\ddagger}$ Ovarian carcinosarcoma, transitional cell cancer, maledifferentatum and psammomacarcinoma of ovary.

receiving chemotherapy within the last 14 days of life, more than one emergency room visit within the last 30 days of life, more than one hospital admission in the 
last 30 days of life, more than 14 days spent admitted to the hospital in the last 30 days of life, intensive care unit admission during the last 30 days of life and death in hospital [8].

According to the Finnish legislation, no ethical approval from official ethics committee or consent from the patient is mandatory for a register-based study. The Finnish law (Medical Research Act 1999/488, 2004/295, 2010/794) states medical research requiring the approval of an appropriate ethics committee as following: research involving intervention in the integrity of a person, human embryo or human foetus for the purpose of increasing knowledge or the nature of diseases in general. Register based scientific research is stated also in the Act on the Openness of Government Activities (621/1999) and Personal Data Act (523/1999). According to these Acts the administrative approval by the participating hospital is adequate which in this case has been received.

Descriptive statistics were used to characterize the study cohort as median with range or frequency and percentage. A chi-squared test was used in analysing frequency tables. Survival analyses were based on Kaplan-Meier method and log rank-test was used to analyse OS, PFS and TFI times. Median survival time with $95 \%$ confidence intervals was expressed and survival curves were plotted. Statistically significant results were considered with a value of $\mathrm{p}<0.05$. SPSS for Windows (version 27, IBM Corp., Armonk, N.Y., USA) was used for statistical analyses.

\section{Results}

\section{Characteristics of the patients}

Most of the patients $(80 \%, \mathrm{n}=151)$ had advanced stage III or IV disease at the time of diagnosis. A majority of them $(85 \%, \mathrm{n}=160)$ had underwent primary or secondary cytoreductive surgery. Details of the characteristics of the patients are summarized in Table 1.

\section{Chemotherapy lines}

When all patients were included in analyses, the median number of the received chemotherapy lines was two (mean 3, range $1-14$ ). Of the patients, $38 \%$ $(n=72)$ had received four or more lines of chemotherapy at the end of follow up. As Figure 1 shows, each patient received a platinum-based regimen as the first-line regimen. The most common chemotherapeutic regimen for primary disease was a paclitaxel-carboplatin combination $(74 \%, \mathrm{n}=140)$, followed by single-carboplatin $(16 \%, \mathrm{n}=31)$ and a combination of docetaxel and carboplatin $(6 \%, n=11)$. In subsequent treatment lines, the distribution of chemotherapy regimens was more diverse.

\section{$O S$ and PFS}

Altogether 189 women with ovarian cancer, fallopian tube or primary peritoneal cancer were included in this analysis. Recurrence occurred in 116 (61\%) patients (Table 1). The median OS was 61 (95\% CI 47 - 75) months and median PFS was 21 (95\% CI 16 - 26) months. Five-year OS and PFS were $49 \%$ and $18 \%$, 


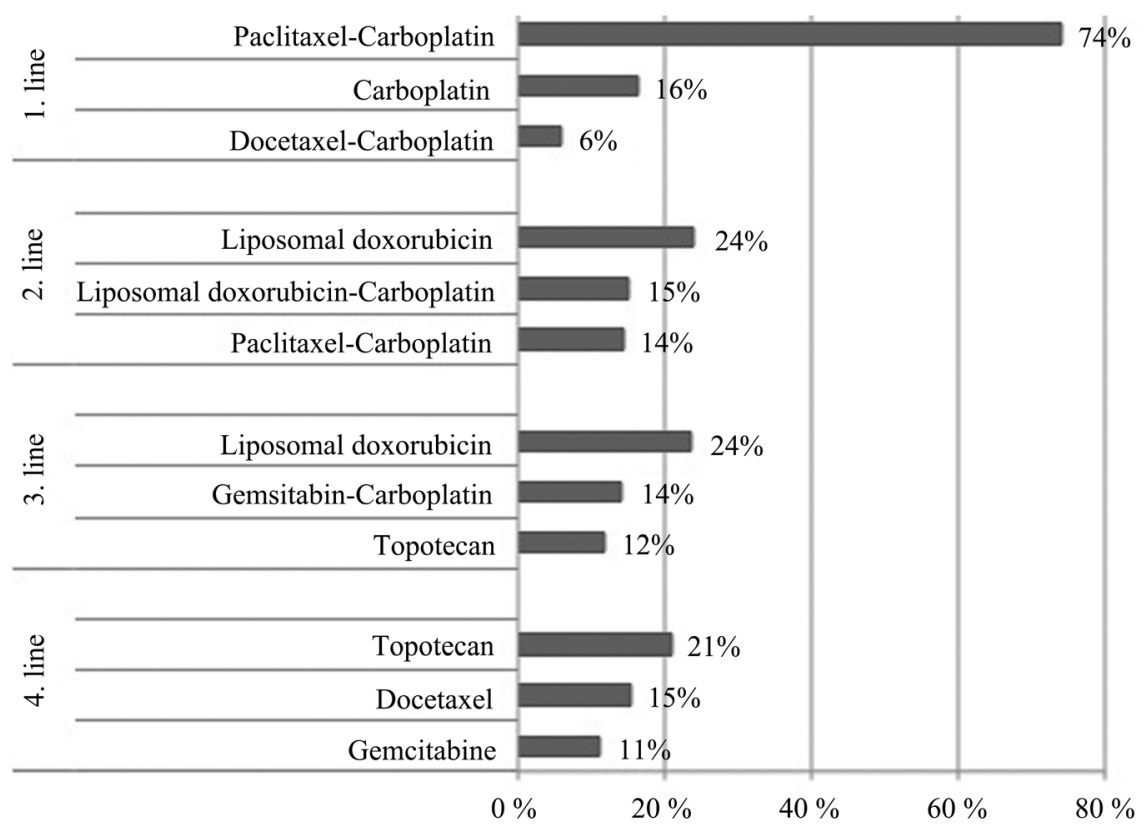

Figure 1. The most common chemotherapy regimens used for patients until the fourth line.

respectively. In platinum-sensitive patients median OS was 94 (95\% CI 71 - 117) months, in semi-sensitive patients 44 (95\% CI 19 - 69) months, and in platinum-resistant patients median OS was 35 (95\% CI 23 - 48) months.

Figure $2(\mathrm{~A})$ shows that median OS was significantly higher after primary treatment compared to subsequent relapses $(\mathrm{p}<0.001)$. Median OS after the first, second, third and 4+ relapse was 38.0 (95\% CI 33.7 - 42.3), 29.0 (95\% CI 21.4 - 36.6), 27.0 (95\% CI 19.2 - 34.8) and 19.0 (95\% CI 13.5 - 24.5) months, respectively. Similarly, median PFS was significantly longer after primary disease compared to subsequent relapses $(\mathrm{p}<0.001)$. Median PFS after the first, second, third and 4+ relapse was 15.0 (95\% CI 13.7 - 16.3), 13.0 (95\% CI 12.0 - 14.0), 15.0 (95\% CI 13.1 - 16.9) and 14.0 (95\% CI 8.3 - 19.7) months, respectively (Figure 2(B)).

\section{TFIs}

As shown in Figure 3(A), TFIs shortened significantly after the first relapse and subsequent number of chemotherapy lines $(p<0.001)$. After the first, second, third, fourth and 5+ chemotherapy lines median TFI was 12.0 (95\% CI 9.2 - 14.8), 4.0 (95\% CI 2.2 - 5.8), 3.0 (95\% CI 1.4 - 4.6), 3.0 (95\% CI 1.8 - 4.2) and 1.0 (95\% CI 0.4 - 1.6) months, respectively. The differences in TFIs were also seen when comparing platinum sensitive, semi-sensitive and platinum resistant patients. After the first, second, third, fourth and 5+ chemotherapy lines median TFI in platinum sensitive patients was 32 (95\% CI 22.9 - 41.1), 11 (95\% CI5.7 16.3), 4 (95\% CI 0.0 - 11.2), 6 (95\% CI 2.5 - 9.5) and 3 (95\% CI 0.1 - 5.9) months respectively, $(\mathrm{p}<0.001)$. In semi-sensitive patients median TFI was $8(95 \% \mathrm{CI}$ 6.4 - 9.6), 3 (95\% CI 1.2 - 4.8), 6 (95\% CI 2.3 - 9.7), 6 (95\% CI 3.3 - 8.8) and 1 $(95 \%$ CI $0.0-2.4)$ months respectively $(\mathrm{p}<0.001)$ and in platinum resistant 

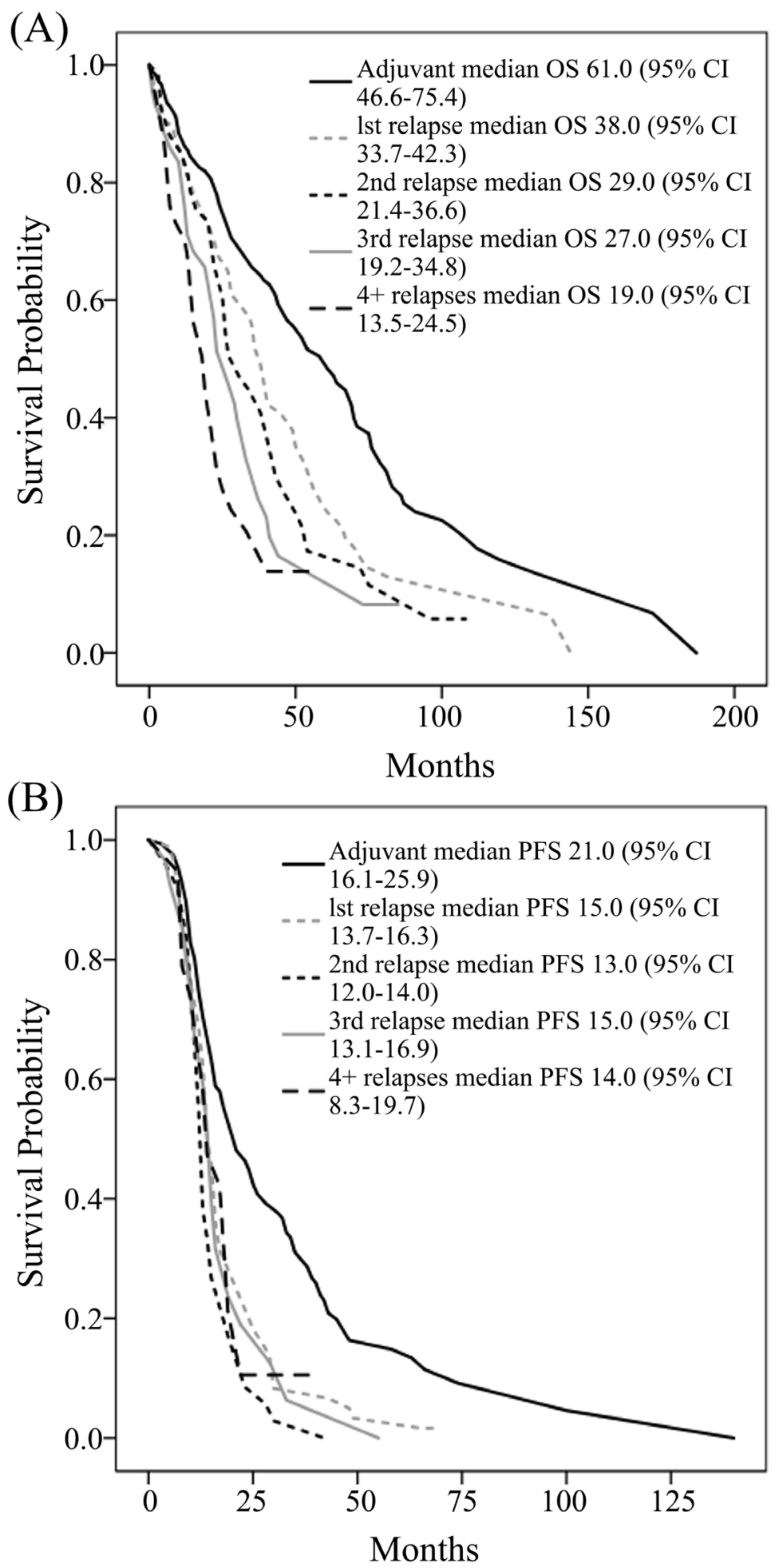

Figure 2. (A) OS after primary disease and after subsequent relapses $1-4+,(\mathrm{p}<0.001)$. (B) PFS after primary disease and after subsequent relapses $1-4+,(\mathrm{p}<0.001)$.

patients median TFI was 4 (95\% CI 2.8 - 5.2), 3 (95\% CI 1.5 - 4.5), 2 (95\% CI 0.7 - 3.3), 2 (95\% CI 1.1 - 2.9) and 1 (95\% CI 0.0 - 2.0) months respectively ( $\mathrm{p}=$ 0.003 ) (Figure 3). 


\section{Overall TFI}

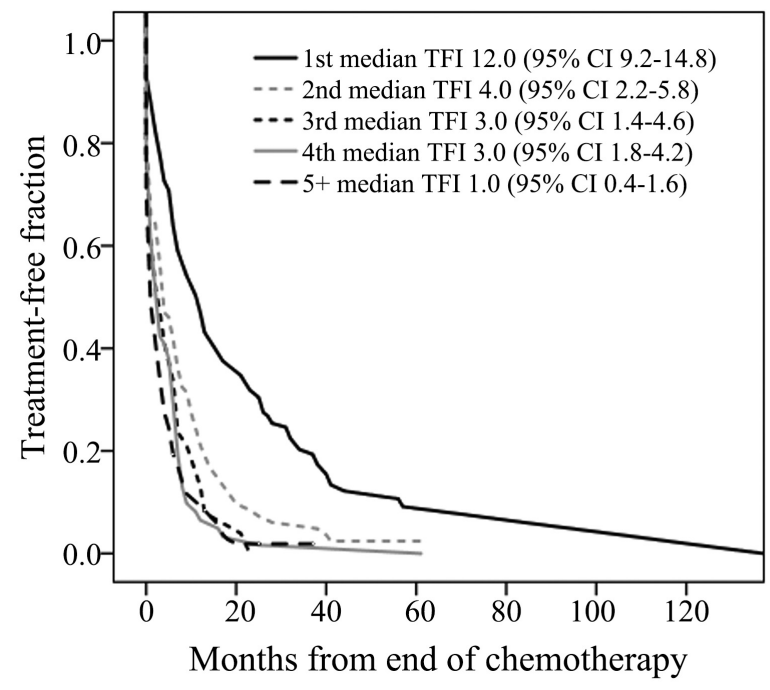

(A)

First recurrence 6-12 months after primary treatment

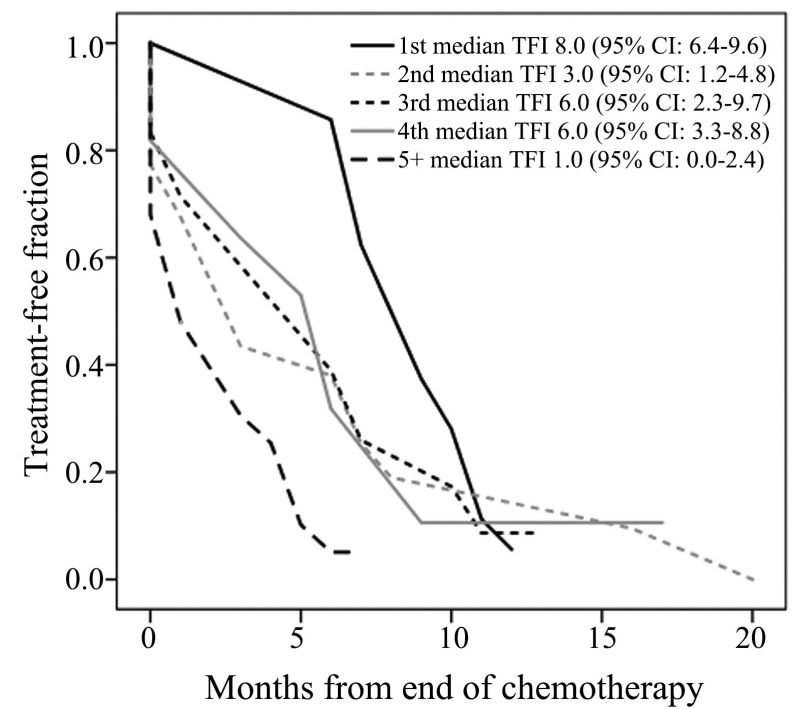

(C)
First recurrence under 6 months after primary treatment

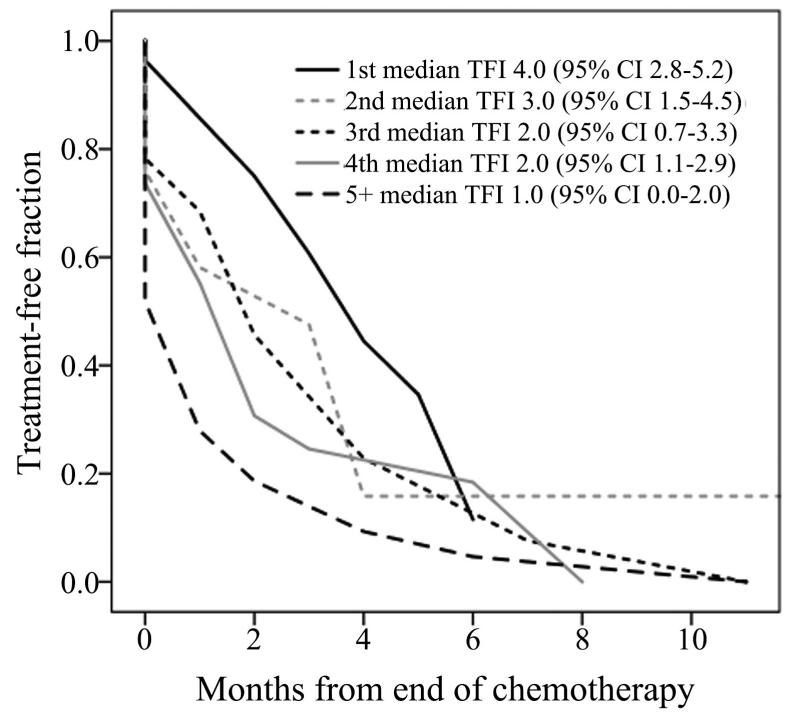

(B)

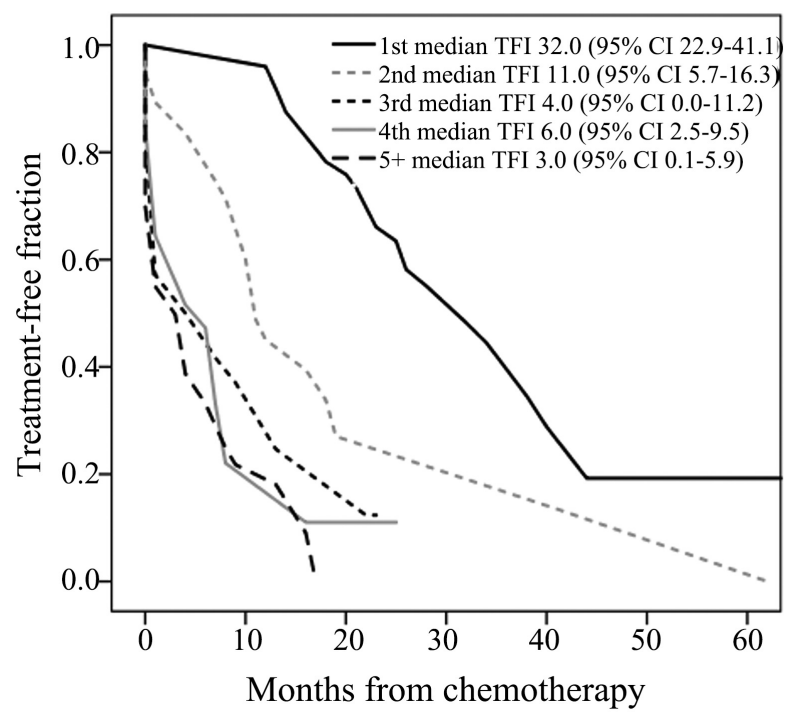

(D)

Figure 3. (A) TFIs after subsequent number of chemotherapy lines in all patients, $(p<0.001)$. (B) TFIs in platinum resistant patients, $(p=0.003)$. (C) TFIs in semi-sensitive patients, $(p<0.001)$. (D) TFIs in platinum sensitive patients, $(p<0.001)$.

\section{Aggressive care and TFI}

We summarized all TFIs of each patient during her total follow up time. It was found that the total proportion of TFI times of the whole follow up time was significantly decreased in those patients that received at least one form of aggressive care at end of their lives compared to those patients without aggressive care at the end of life ( $41 \%$ vs. $55 \%, p=0.004)$ (Figure 4$)$. The last TFI one month or less between the last chemotherapy line and death was associated with at least one form of aggressive care in the last 30 days of life $(\mathrm{p}=0.029)$. 


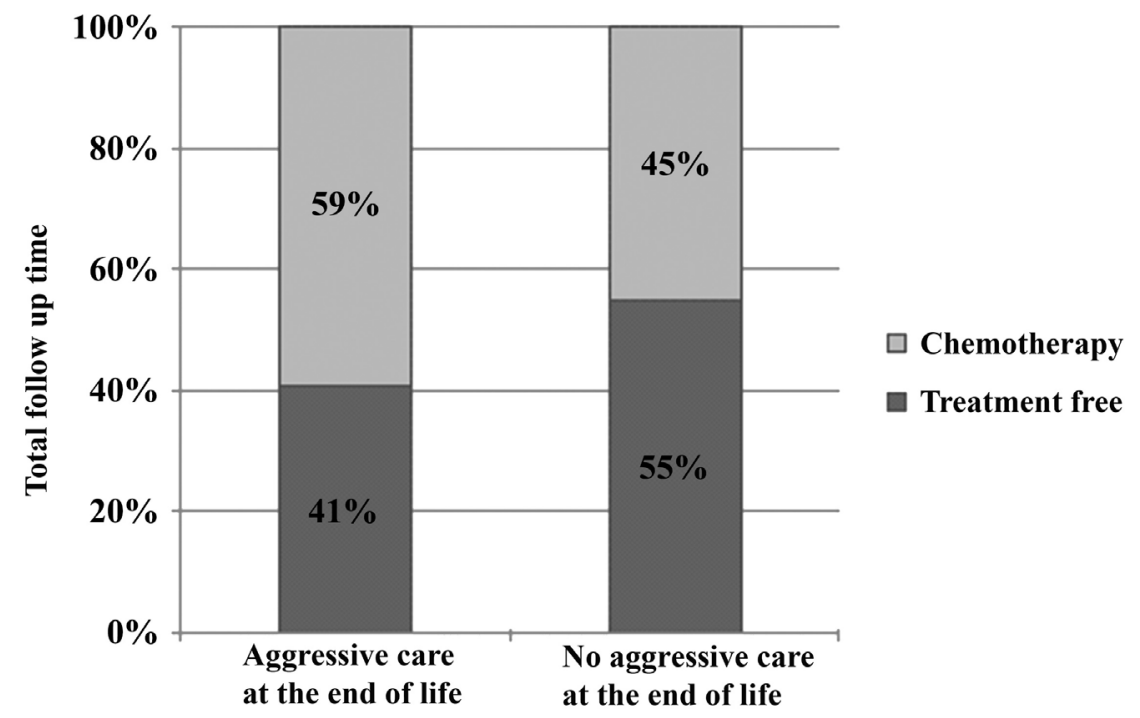

Figure 4. Proportions when patients were treatment free or receiving chemotherapy during their follow-up. A total treatment free time during the follow up was only $41 \%$ if patient had received aggressive care in the last 30 days of life, $(p=0.004)$.

\section{Discussion}

We describe here a single tertiary hospital experience how OS and PFS rates and TFI times behaved after multiple chemotherapy lines. We found that TFIs were significantly shortened after the first relapse when subsequent chemotherapy lines were used. Aggressive care at the end of life was associated with the decreased total treatment free time during the follow-up.

Standards for the first line platinum-based chemotherapy regimens in ovarian cancer are well-established [9]. For very platinum sensitive patients a combination of carboplatin and pegylated liposomal doxorubisin is recommended [10]. However, in later relapses the best and the most effective regimens for the patient are not so clear. Patients in our cohort were treated according to recommendations since each patient received platinum in first line. The second line regimens were platinum-based when the patient was platinum sensitive and in platinum resistant situations pegylated liposomal doxorubicin was used most often. Generally, single antineoplastic agent is referred in patients with platinum resistant disease and poor prognosis. The main goal of the treatment in those patients is preservation of quality of life and avoid toxicities as much as possible.

As mentioned before, there is an unmet need for trials of patients that have received multiple chemotherapy lines [5]. Apart from BRCA status [11], there are no reliable predictors to help selection of the patients who are more likely to respond to three or more lines of chemotherapy. To identify those patients Hoskins et al. [12] describe a model that predicts lack of benefit by the length of an interval between two preceding relapses, in which under 12 months from diagnosis to second relapse, under six months from first to third relapse and second to fourth relapse predict a survival of six months or less. A study by Hanker et al. based on three clinical ovarian cancer clinical trials [13] showed 
that a maximum of three lines of subsequent relapse treatment seems to be beneficial according to OS for patients with recurrent ovarian cancer. Recently, a large GCIG Symptom Benefit Study reported that baseline HRQL (health-related quality of life) combined with clinicopathological prognostic factors like physical, cognitive and social functions and abdominal/GI symptoms is able to assist clinician in decision making of further cancer treatments [14].

When evaluating the effectiveness of non-curative treatments, OS is not only parameter of describing benefit. TFI is traditionally used to divide patients whether they are platinum sensitive or resistant [9]. In this study, we used TFI to describe the time when patients are without cancer-targeted treatment during their follow-up after serial chemotherapy lines. The correlation between longer TFI and better health-related quality of life has been reported in the treatment of multiple myeloma [15]. In ovarian cancer, TFIs has been shown to decrease significantly after serial chemotherapy treatments [3] and that was also noticed by us. In our study TFI was one month or less after the fifth relapse suggesting that by that time point at the latest further chemotherapy might be rather harmful than beneficial to patients.

When comparing platinum sensitive, semi-sensitive and platinum resistant patients we expectedly saw that TFIs after the first and second treatment line in platinum sensitive patients were notably higher, than in platinum resistant and semi-sensitive patients. Patients that experienced platinum resistant disease after the first relapse had very poor prognosis in general. According to TFIs, chemotherapies should be avoided after second-line treatments in those patients since TFIs were only two months or less.

Patients that received at least one form of aggressive care in the last 30 days of life had decreased overall TFI compared to those without aggressive care. More specifically, patients without aggressive care at the end of life were more than half of their follow up time without cancer targeted medication compared to those patients with aggressive care and $41 \%$ of the follow up time without chemotherapies. In many cases the aim of chemotherapy is to palliate symptoms. In a study by Friedlander et al. [16] in patients with recurrent ovarian cancer and palliative chemotherapy, half of the patients gained improvement regarding their symptoms, but many progressed rapidly. Thus, measures to document symptoms, adverse events and quality of life might be useful when deciding to further continue chemotherapy in recurrent settings.

There are several limitations to the present study. Data was collected from a single university hospital thus findings cannot be generalized to other institutions. However, this study describes typical practice in our institution with a reasonable patient cohort. In this study we focused on chemotherapy regimens, but it is important to notice that currently targeted therapies like maintenance bevacizumab [17], PARP inhibitors [18] [19] and immunotherapies [20] are new possibilities to the patients and have impact on reporting TFIs. As a limitation, our data lack the quality of life measurements. 


\section{Conclusion}

In conclusion, we present that patients with recurrent ovarian cancer are commonly treated with multiple chemotherapy lines. TFIs decrease after the first relapse; especially platinum resistant patients have notably short TFIs. More attention should be paid to the careful consideration when further chemotherapy treatment is beneficial.

\section{Funding}

This study was supported by The Finnish Medical Foundation and Kuopio University Hospital VTR grant.

\section{Conflicts of Interest}

The authors declare no conflicts of interest regarding the publication of this paper.

\section{References}

[1] Engholm, G., Ferlay, J., Christensen, N., Hansen, H., Hertzum-Larsen, R., Johannesen, T., et al. (2017) Cancer Incidence, Mortality, Prevalence and Survival in the Nordic Countries, Version 8.0 (20.12.2017). Association of the Nordic Cancer Registries. Danish Cancer Society. http://www.ancr.nu

[2] Timmermans, M., Sonke, G.S., Van de Vijver, K.K., van der Aa, M.A. and Kruitwagen, R.F.P.M. (2018) No Improvement in Long-Term Survival for Epithelial Ovarian Cancer Patients: A Population-Based Study between 1989 and 2014 in the Netherlands. European Journal of Cancer, 88, 31-37. https://doi.org/10.1016/j.ejca.2017.10.030

[3] Eng, K.H., Hanlon, B.M., Bradley, W.H. and Szender, J.B. (2015) Prognostic Factors Modifying the Treatment-Free Interval in Recurrent Ovarian Cancer. Gynecologic Oncology, 139, 228-235. https://doi.org/10.1016/j.ygyno.2015.09.011

[4] Bailey, C.H., Jameson, G., Sima, C., Fleck, S., White, E., Von Hoff, D.D., et al. (2012) Progression-Free Survival Decreases with Each Subsequent Therapy in $\mathrm{Pa}$ tients Presenting for Phase I Clinical Trials. Journal of Cancer, 3, 7-13. https://doi.org/10.7150/jca.3.7

[5] Wilson, M.K., Pujade-Lauraine, E., Aoki, D., Mirza, M.R., Lorusso, D., Oza, A.M., et al. (2017) 5th Ovarian Cancer Consensus Conference of the Gynecologic Cancer InterGroup: Recurrent Disease. Annals of Oncology, 28, 727-732. https://doi.org/10.1093/annonc/mdw663

[6] Yoshihama, T., Chiyoda, T., Kataoka, F., Nomura, H., Iguchi, Y., Hashimoto, S., et al. (2015) Effectiveness of Third-Line Chemotherapy in Recurrent Ovarian Cancer Patients. European Journal of Gynaecological Oncology, 36, 424-427.

[7] Sallinen, H., Rintanen, V., Keski-Nisula, L. and Anttila, M. (2021) Evaluation of Ovarian Cancer Care at the End of Life in a Single Tertiary Hospital. Journal of Cancer Therapy, 12, 86-96. https://doi.org/10.4236/jct.2021.122010

[8] Anonymous National Voluntary Consensus Standards for Quality of Cancer Care. http://www.qualityforum.org/Publications/2009/05/National Voluntary Consensus Standards for Quality of Cancer Care.aspx

[9] The ICON and AGO Collaborators (2003) Paclitaxel plus Platinum-Based Chemo- 
therapy versus Conventional Platinum-Based Chemotherapy in Women with Relapsed Ovarian Cancer: The ICON4/AGO-OVAR-2.2 Trial. The Lancet, 361, 20992106. https://doi.org/10.1016/S0140-6736(03)13718-X

[10] Mahner, S., Meier, W., du Bois, A., Brown, C., Lorusso, D., Dell'Anna, T., et al. (2015) Carboplatin and Pegylated Liposomal Doxorubicin versus Carboplatin and Paclitaxel in Very Platinum-Sensitive Ovarian Cancer Patients: Results from a Subset Analysis of the CALYPSO Phase III Trial. European Journal of Cancer, 51, 352 358. https://doi.org/10.1016/j.ejca.2014.11.017

[11] Ledermann, J., Harter, P., Gourley, C., Friedlander, M., Vergote, I., Rustin, G., et al., (2014) Olaparib Maintenance Therapy in Patients with Platinum-Sensitive Relapsed Serous Ovarian Cancer: A Preplanned Retrospective Analysis of Outcomes by BRCA Status in a Randomised Phase 2 Trial. The Lancet Oncology, 15, 852-861. https://doi.org/10.1016/S1470-2045(14)70228-1

[12] Hoskins, P.J. and Le, N. (2005) Identifying Patients Unlikely to Benefit from Further Chemotherapy: A Descriptive Study of Outcome at Each Relapse in Ovarian Cancer. Gynecologic Oncology, 97, 862-869. https://doi.org/10.1016/j.ygyno.2005.03.022

[13] Hanker, L.C., Loibl, S., Burchardi, N., Pfisterer, J., Meier, W., Pujade-Lauraine, E., et al. (2012) The Impact of Second to Sixth Line Therapy on Survival of Relapsed Ovarian Cancer after Primary Taxane/Platinum-Based Therapy. Annals of Oncology, 23, 2605-2612. https://doi.org/10.1093/annonc/mds203

[14] Roncolato, F.T., O’Connell, R.L., Joly, F., Lanceley, A., Hilpert, F,. Buizen, L., et al. (2020) Predictors of Progression Free Survival, Overall Survival and Early Cessation of Chemotherapy in Women with Potentially Platinum Sensitive (PPS) Recurrent Ovarian Cancer (ROC) Starting Third or Subsequent Line $(\geq 3)$ Chemotherapy-The GCIG Symptom Benefit Study (SBS). Gynecologic Oncology, 156, 45-53. https://doi.org/10.1016/j.ygyno.2019.10.001

[15] Acaster, S., Gaugris, S., Velikova, G., Yong, K. and Lloyd, A. (2013) Impact of the Treatment-Free Interval on Health-Related Quality of Life in Patients with Multiple Myeloma: A UK Cross-Sectional Survey. Supportive Care in Cancer, 21, 599-607. https://doi.org/10.1007/s00520-012-1548-y

[16] Friedlander, L.M., Stockler, T.M., O'connell, T.R., Voysey, T.M., Oza, T.A., Gillies, T.K., et al. (2014) Symptom Burden and Outcomes of Patients with Platinum Resistant/Refractory Recurrent Ovarian Cancer: A Reality Check: Results of Stage 1 of the Gynecologic Cancer Intergroup Symptom Benefit Study. International Journal of Gynecological Cancer, 24, 857-864. https://doi.org/10.1097/IGC.0000000000000147

[17] Pfisterer, J., Shannon, C.M., Baumann, K., Rau, J., Harter, P., Joly, F., et al. (2020) Bevacizumab and Platinum-Based Combinations for Recurrent Ovarian Cancer: A Randomised, Open-Label, Phase 3 Trial. The Lancet Oncology, 21, 699-709. https://doi.org/10.1016/S1470-2045(20)30142-X

[18] Mirza, M.R., Monk, B.J., Herrstedt, J., Oza, A.M., Mahner, S., Redondo, A., et al. (2016) Niraparib Maintenance Therapy in Platinum-Sensitive, Recurrent Ovarian Cancer. The New England Journal of Medicine, 375, 2154-2164. https://doi.org/10.1056/NEJMoa1611310

[19] Pujade-Lauraine, E., Ledermann, J.A., Selle, F., Gebski, V., Penson, R.T., Oza, A.M., et al. (2017) Olaparib Tablets as Maintenance Therapy in Patients with PlatinumSensitive, Relapsed Ovarian Cancer and a BRCA1/2 Mutation (SOLO2/ENGOTOv21): A Double-Blind, Randomised, Placebo-Controlled, Phase 3 Trial. The Lancet Oncology, 18, 1274-1284. https://doi.org/10.1016/S1470-2045(17)30469-2 
[20] Matulonis, U.A., Shapira-Frommer, R., Santin, A.D., Lisyanskaya, A.S., Pignata, S., Vergote, I., et al. (2019) Antitumor Activity and Safety of Pembrolizumab in Patients with Advanced Recurrent Ovarian Cancer: Results from the Phase II KEYNOTE-100 Study. Annals of Oncology, 30, 1080-1087. https://doi.org/10.1093/annonc/mdz135 